Acta Crystallographica Section D

Biological

Crystallography

ISSN 0907-4449
Kevin S. Keating ${ }^{a}$ and Anna Marie Pyle ${ }^{a, b, c *}$

${ }^{a}$ Department of Molecular, Cellular and Developmental Biology, Yale University, New Haven, CT 06511, USA, bepartment of Chemistry, Yale University, New Haven, CT 06511, USA, and ${ }^{\mathrm{C}}$ Howard Hughes Medical Institute, USA

Correspondence e-mail: anna.pyle@yale.edu

\title{
RCrane: semi-automated RNA model building
}

RNA crystals typically diffract to much lower resolutions than protein crystals. This low-resolution diffraction results in unclear density maps, which cause considerable difficulties during the model-building process. These difficulties are exacerbated by the lack of computational tools for RNA modeling. Here, RCrane, a tool for the partially automated building of RNA into electron-density maps of low or intermediate resolution, is presented. This tool works within Coot, a common program for macromolecular model building. RCrane helps crystallographers to place phosphates and bases into electron density and then automatically predicts and builds the detailed all-atom structure of the traced nucleotides. RCrane then allows the crystallographer to review the newly built structure and select alternative backbone conformations where desired. This tool can also be used to automatically correct the backbone structure of previously built nucleotides. These automated corrections can fix incorrect sugar puckers, steric clashes and other structural problems.

\section{Introduction}

In recent years, RNA crystal structures have contributed greatly to the understanding of numerous cellular processes (Ban et al., 2000; Batey et al., 2004; Selmer et al., 2006). However, these structural studies are complicated by the fact that RNA crystals normally diffract to lower resolutions than protein crystals (Keating \& Pyle, 2010). This low-resolution diffraction results in unclear electron-density maps, which frequently lead to errors in the structure-determination process. For protein crystallography, numerous tools exist for automated and partially automated model building (Cowtan, 2006; Langer et al., 2008; Terwilliger, 2003); however, computational tools for RNA crystallography are only beginning to emerge. These tools aid in detecting (Chen et al., 2010) and correcting (Wang et al., 2008) errors in crystallographic models, interpreting electron density (Gruene \& Sheldrick, 2011) and classifying specific substructures (Sarver et al., 2008; Wadley et al., 2007), but few tools are available to aid in constructing the initial crystallographic model (Hattne \& Lamzin, 2008).

Here, we present RCrane (RNA constructed using rotameric nucleotides), a tool for semi-automated model building of RNA into electron-density maps of low or intermediate resolution. RCrane first helps crystallographers to place phosphate and base atoms into electron density and then automatically predicts and builds the all-atom structure of the traced nucleotides. The prediction and building protocols are based on techniques that have previously been shown to produce highly accurate structures (Keating \& Pyle, 2010), but
Received 20 December 2011 Accepted 25 April 2012 
the computer-assisted phosphate- and base-placement algorithms are newly developed and thoroughly tested below.

RCrane takes advantage of the recently developed consensus backbone conformer library (Richardson et al., 2008). This conformer library enumerates roughly 50 discrete configurations for the RNA backbone and is comparable to the side-chain rotamer libraries frequently used in protein model building (Lovell et al., 2000). It is important to note that the backbone conformer library divides the RNA backbone into suites rather than nucleotides, where each suite spans two sugars and the intervening phosphate (Fig. 1). The conformers are given two character names, such as $1 \mathrm{a}$, where the first character represents the initial $\delta, \varepsilon$ and $\zeta$ torsions and the second character represents the $\alpha, \beta, \gamma$ and $\delta$ torsions. Additionally, any two adjacent suites overlap by one sugar (and the associated $\delta$ torsion), and therefore the ending pucker of one suite must be identical to the leading sugar pucker of the following suite.

RCrane also utilizes a modified pseudotorsional system (Keating \& Pyle, 2010) that can classify RNA structure based solely on the phosphate and $\mathrm{Cl}^{\prime}$ coordinates. These atoms can be accurately located in electron-density maps even when working at low or intermediate resolution. Phosphates are clearly visible owing to their high electron density. Conversely, the $\mathrm{Cl}^{\prime}$ atom is difficult to locate directly; however, its location can be easily and uniquely determined from the coordinates of the nucleoside base (Keating \& Pyle, 2010), which can be accurately located in density owing to its large size and rigidity. This minimal representation of RNA also holds potential for noncrystallographic applications such as molecular dynamics, where all-atom representations are not always possible owing to constraints in computing power (Cao \& Chen, 2005; Jonikas et al., 2009).

\section{Program features}

The functionality of RCrane can be divided into a number of major features. The initial model building consists of three main steps: backbone tracing, conformer prediction and coordinate calculation. Backbone tracing is carried out interactively using input from the crystallographer. The remaining two steps, however, are fully automated. After the initial model has been built, it may be reviewed and modified during alternate-conformer selection. RCrane also allows the crystallographer to modify an existing structure. This feature can be used to correct structures built without RCrane, or it can be used to revise structures after crystallographic refinement. Additionally, RCrane works within Coot, a common program for macromolecular model building (Emsley et al., 2010), and is designed to be intuitively usable by crystallographers familiar with the Coot interface.

\subsection{Backbone tracing}

When constructing an initial model with RCrane, the first step is to build a backbone trace (Fig. 2a), in which the phosphate and base atoms are placed into the electron density.
In RCrane this step is performed interactively. The plugin provides suggestions for atom locations, but the crystallographer must play an active role in the tracing process. The backbone trace begins with locating a single phosphate. When the user begins the trace, the plugin searches for all potential phosphate locations within $10 \AA$ of the screen center (see $\S 3$ ). The phosphate closest to the screen center is initially selected, and the user may accept this phosphate location, select an alternate location, or manually adjust the phosphate coordinates.

Once the user accepts a phosphate location, RCrane will automatically trace a potential location for the next base and phosphate (see \$3) and display these traced atoms. After the traced atoms are displayed, the user may accept the default coordinates, select an alternate base or phosphate location, or manually adjust the base or phosphate locations. The user must ensure that the traced atoms truly represent a base and phosphate, as density from metal ions can sometimes appear to be similar to density from phosphate groups. After the user accepts a set of coordinates, the next nucleotide is traced in a similar fashion. This process is repeated until the user has traced all of the desired connected nucleotides. Note that here 'next' may refer to either the $5^{\prime}$ or $3^{\prime}$ nucleotide depending on the directionality of the trace. This directionality is set by the user at the start of the trace.

\subsection{Conformer prediction}

After the backbone trace has been completed, RCrane must predict an appropriate conformer for each suite to be built. This step is performed automatically and does not require any user input. The prediction process is carried out as previously described (Keating \& Pyle, 2010); however, six additional conformers are now considered during prediction (see $\$ 3$ ). For each suite, this prediction assigns a score to each conformer. These scores sum to one for each suite and thus approximate a percentage likelihood for the given conformer. All scores are displayed during alternate-conformer selection (see below).

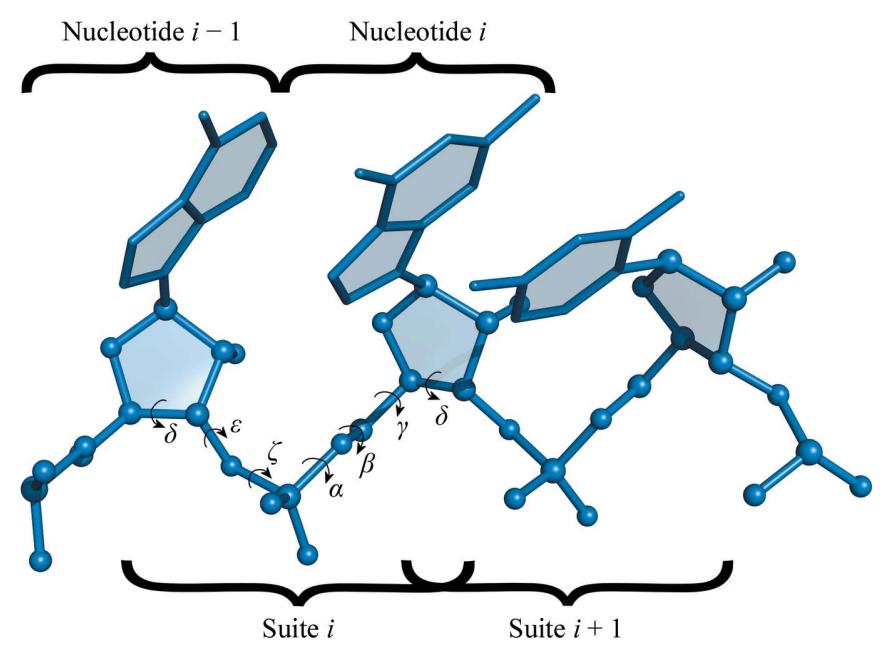

Figure 1

The RNA backbone, with suite and nucleotide divisions indicated. The backbone torsions belonging to suite $i$ are also shown. 


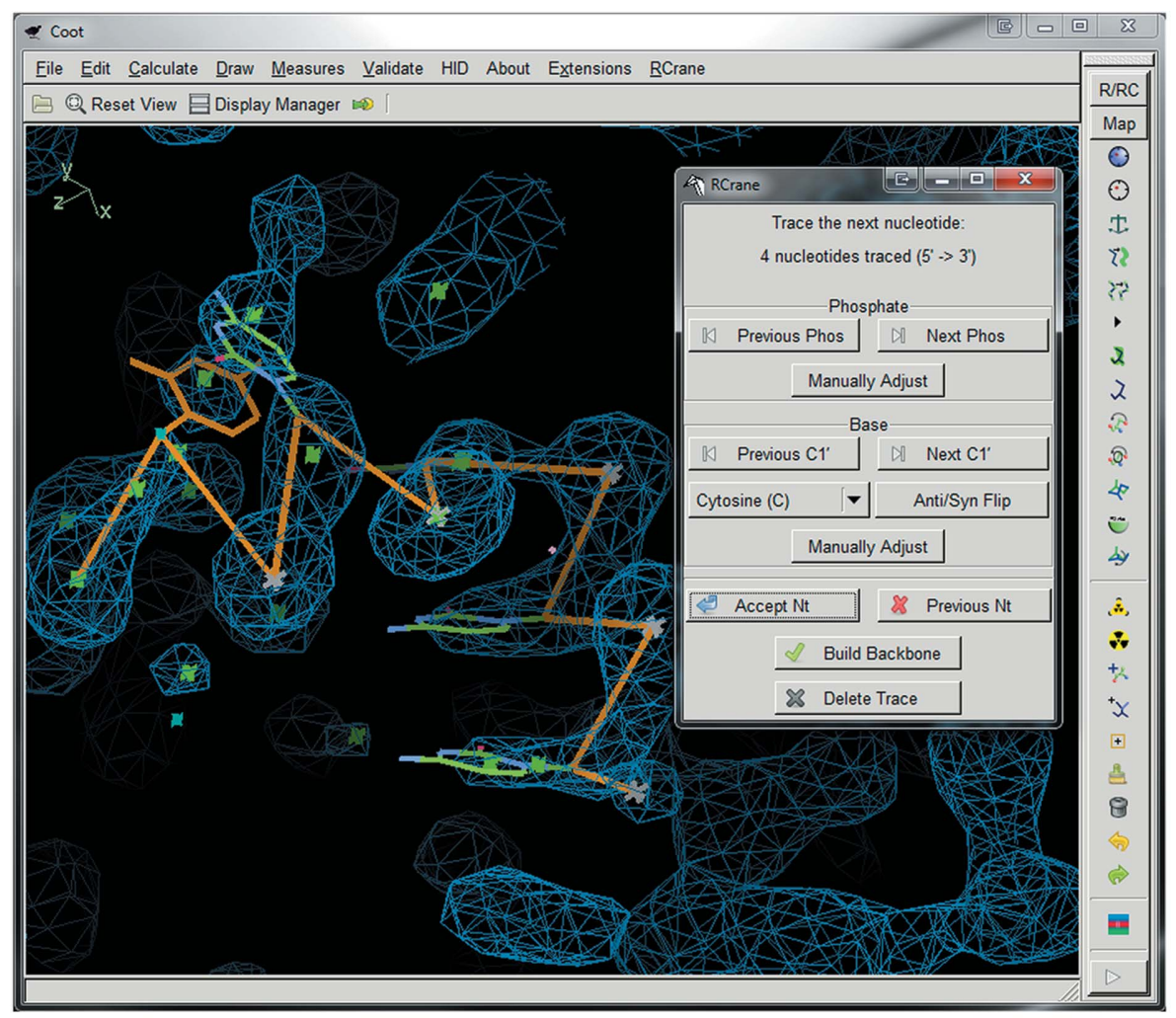

(a)

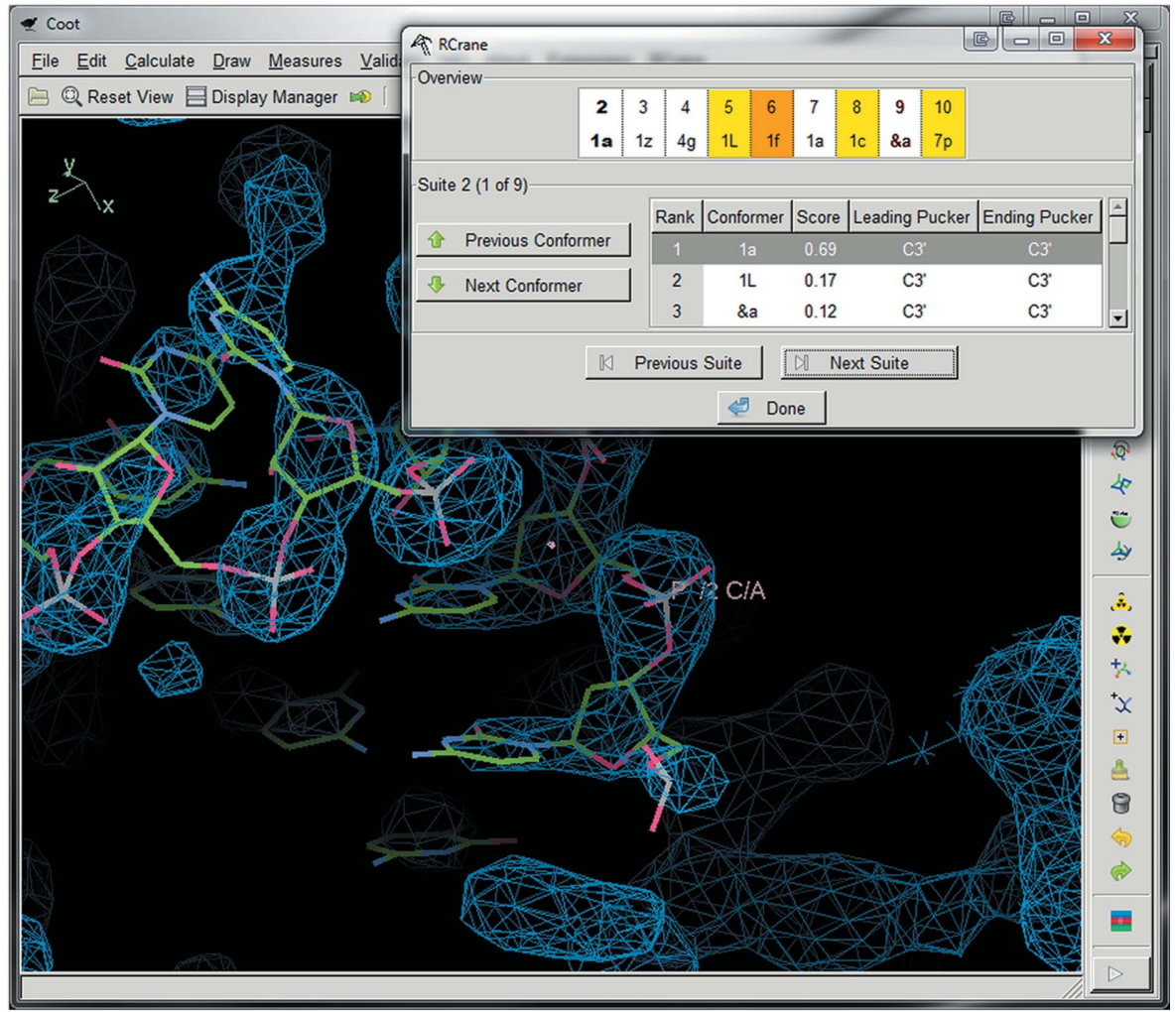

(b)

\section{Figure 2}

The RCrane interface. (a) RCrane assists the crystallographer in tracing phosphates and bases in the electron-density map. Here, the traced nucleotides are shown in green and orange, with the nucleotide that is currently being traced shown entirely in orange. The RCrane window on the right allows the user to select alternate phosphate and base locations. (b) After the backbone has been traced, RCrane automatically builds an all-atom model of the traced nucleotides. The user may then review the newly built structure and select alternate conformers where desired.

\subsection{Coordinate calculation}

After conformer prediction, atomic coordinates must be calculated that match both the predicted conformer and the previously determined phosphate and base locations. As with conformer prediction, this step is performed automatically and does not require any user input. Coordinate calculation takes advantage of the minimization functions built into Coot. As a result, coordinate calculation is now approximately one order of magnitude faster than previously reported (Keating \& Pyle, 2010). Additionally, the minimization procedure now incorporates information about the electron density. The resulting coordinates are still primarily determined using the predicted conformers and the traced phosphate and base locations; however, the inclusion of an electron-density term in the minimization helps to ensure that the calculated coordinates fit the density map as closely as possible (Fig. 3).

\subsection{Alternate-conformer selection}

After conformer prediction and coordinate calculation are complete, the user is presented with the newly built nucleotides and given the opportunity to review the structure (Fig. 2b). The current conformers for each of the built suites are shown in the review window. This review window is colored using a 'traffic-light' color scheme, in which suites that should be reviewed by the crystallographer are highlighted in yellow, orange or red depending on the degree of uncertainty for that suite. This uncertainty may arise from either the conformer-prediction or the coordinatecalculation step. Uncertainty in conformer prediction occurs when the most likely and second most likely conformers are predicted to have similar likelihoods. Uncertainty in coordinate calculation occurs when the minimization procedure was unable to find an ideal match between the predicted conformer and the traced phosphate and base coordinates. Note that this 'traffic-light' color scheme is already used to present refinement results within Coot (Emsley et al., 2010). 
Thus, RCrane and Coot present a consistent user interface for reviewing structural information.

This review window presents a list of all conformers and their conformer-prediction scores for the current suite (see above). This list is sorted by score and therefore presents likely alternate conformers at the top. From this window, the user can click on any alternate conformer and RCrane will automatically rebuild the affected nucleotides and display the results. If the alternate conformer requires a change in sugar pucker, adjacent suites will also be rebuilt to account for this pucker change. (Adjacent suites overlap by one sugar, so changing a sugar pucker in one suite implicitly changes a sugar pucker in an adjacent suite.) The review window thus allows the crystallographer to quickly examine a number of alternate conformers and select the most appropriate one.

\subsection{Rotamerization}

The previous features have been discussed in the context of building a new structure into electron density. However, rotamerization allows the user to correct or improve a region of an existing structure. This feature works similarly to the refine zone or regularize zone options in Coot, which carry out real-space refinement or geometry minimization on a specified region of a protein or nucleic acid structure. With rotamerization, the user first specifies a contiguous stretch of nucleotides. RCrane then uses the existing phosphate and base coordinates to carry out conformer prediction, coordinate calculation and alternate-conformer selection. This rebuilds the specified nucleotides and will typically correct errors in the backbone structure such as incorrect sugar puckers or steric clashes arising from incorrect backbone conformations.

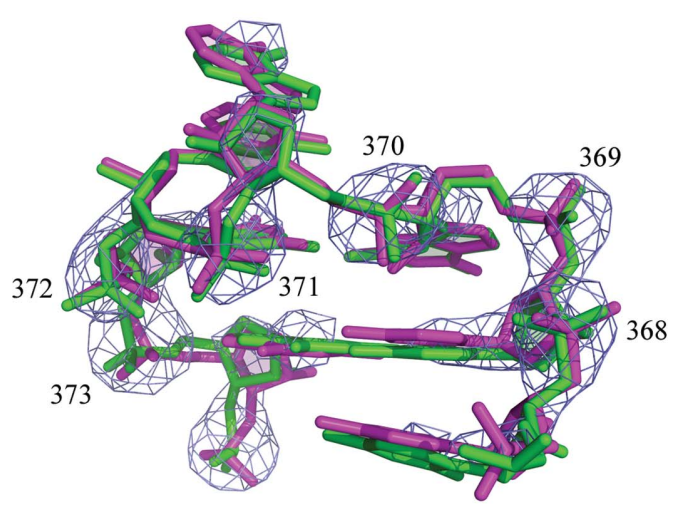

(a)

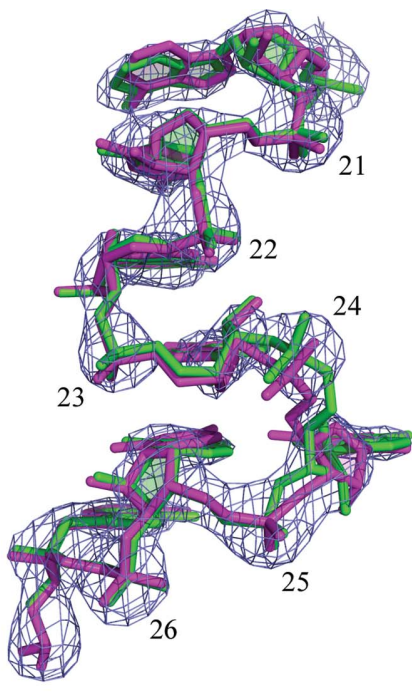

(b)
Figure 3

Coordinates built using RCrane are highly accurate. A comparison of models built using RCrane (magenta) and published coordinates (green) is shown. Suite numbers are as indicated. Note that the structures built using RCrane have not yet undergone crystallographic refinement. (a) The GANC tetraloop from the group II intron (Toor et al., 2010). This structure was built into the $3.1 \AA$ experimentally phased map (Toor et al., 2008) shown contoured at 3.0 $\sigma$. (b) An S-turn motif from the lysine riboswitch (Garst et al., 2008) built into a $2.8 \AA$ density map shown contoured at $1.8 \sigma$.
This feature is useful when revising a model after a round of crystallographic refinement, as the refined map may provide more precise information about nucleotide structure. Rotamerization also provides a complement to services such as MolProbity (Chen et al., 2010), which excels at detecting problem areas within crystallographic models but cannot offer specific fixes for RNA structures.

\section{Methods}

\subsection{Phosphate picking}

The initial step in an RCrane backbone trace is to compile a list of potential phosphate locations. This list is compiled using a peak-search function within Coot. This function is closely related to the Coot algorithm for water picking (i.e. finding electron density corresponding to water molecules). The phosphate search is carried out by first dividing the map into discrete regions of connected electron density. Each region corresponds to a single connected section of electron density and the size of these discrete regions is entirely dependent upon the map itself and the current contour level. At low contour levels these regions may be tens of ångströms long, while at higher contour levels these regions are typically only several ångströms in size. Within each region, the peak is defined as the point with the highest electron density. All peaks within the map are considered to be potential phosphate locations. This peak search is conducted with maps contoured at $1 \sigma$ to $7 \sigma$ in $0.25 \sigma$ intervals and all duplicate peaks are ignored. This search is carried out only once per map and the results are cached for use in subsequent phosphate searches. When beginning a new backbone trace, all potential phosphate locations within $10 \AA$ of the screen center are shown to the user and the initially selected phosphate is the peak closest to the screen center.

\subsection{Nucleotide tracing}

After the user has selected an initial phosphate, RCrane locates potential coordinates for the next base and phosphate. Here, 'next' and 'previous' are dependent on the direction of the backbone trace. When starting from phosphate $i$ and tracing from $5^{\prime}$ to $3^{\prime}$, 'next' refers to base $i$, sugar $i$ and phosphate $i+1$. When tracing from $3^{\prime}$ to $5^{\prime}$, 'next' refers to base $i-1$, sugar $i-1$ and phosphate $i-1$. Similarly, 'previous' refers to base $i-1$, sugar $i-1$ and phosphate $i-1$ when tracing $5^{\prime}$ to $3^{\prime}$, and to base $i$, sugar $i$ and phosphate $i+1$ when tracing $3^{\prime}$ to $5^{\prime}$.

Phosphate candidates are located as described above and all phosphates within $10 \AA$ of the starting phosphate (phosphate $i$ ) are considered to be 
potential next phosphates. For each potential next phosphate, a potential sugar center is found as follows. Let $\mathrm{P}_{5^{\prime}}$ be the coordinates of the $5^{\prime}$ phosphate (i.e. the current phosphate when tracing $5^{\prime}$ to $3^{\prime}$ or the candidate phosphate when tracing $3^{\prime}$ to $5^{\prime}$ ) and let $\mathrm{P}_{3^{\prime}}$ be the coordinates of the $3^{\prime}$ phosphate (i.e. the candidate phosphate when tracing $5^{\prime}$ to $3^{\prime}$ or the current phosphate when tracing $3^{\prime}$ to $5^{\prime}$ ). A cylindrical coordinate system is then defined with an origin at $\mathrm{P}_{5^{\prime}}$ and a vertical (or cylindrical) axis of $\overrightarrow{\mathrm{P}_{5^{\prime}} \mathrm{P}_{3^{\prime}}}$. The polar (or azimuthal) axis is defined as an arbitrary vector orthogonal to $\overrightarrow{\mathrm{P}_{5^{\prime}} \mathrm{P}_{3^{\prime}}}$ and starting at $\mathrm{P}_{5^{\prime}}$.

Let $\mathrm{G}_{\text {candidate }}$ be the coordinates of the candidate sugar center in this cylindrical coordinate system, defined as $(r, \xi, z)$ (Fig. 4a). The radial component of the sugar-center location, $r$, is given by

$$
r=a_{1} d^{2}+a_{2} d+a_{3},
$$

where $d$ is the distance between the $5^{\prime}$ and $3^{\prime}$ phosphates,

$$
d=\left\|\overrightarrow{\mathrm{P}_{5^{\prime}} \mathrm{P}_{3^{\prime}}}\right\| \text {. }
$$

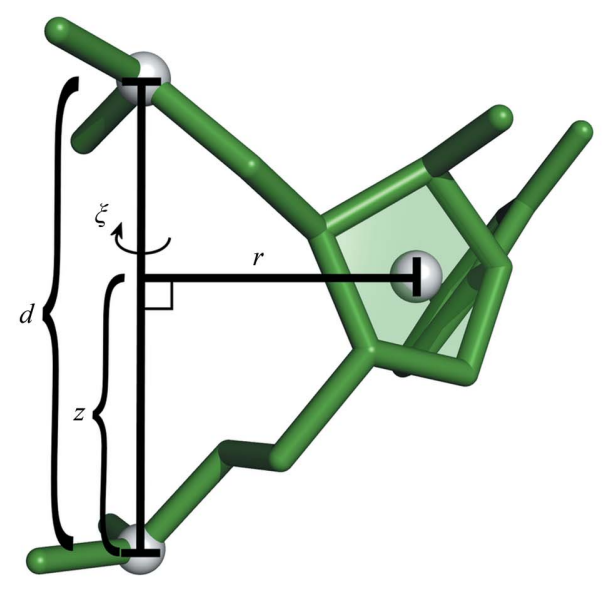

(a)

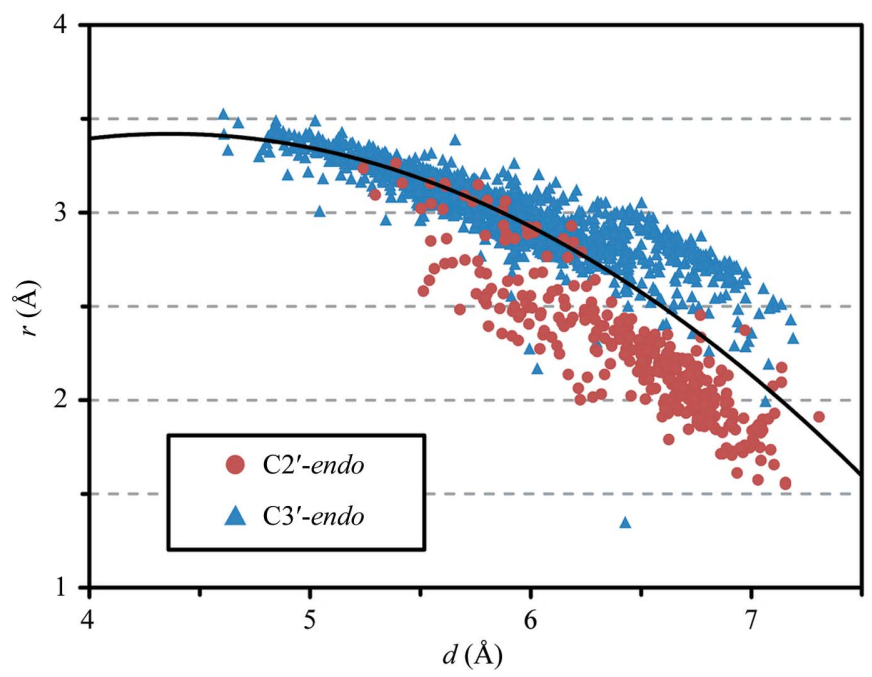

(b)
In (1), $a_{1}=-0.186, a_{2}=1.623$ and $a_{3}=-0.124$. These values were determined empirically using the RNA05 data set (Fig. 4b). The vertical component of the sugar-center location, $z$, is given by

$$
z=b_{1} d+b_{2}
$$

where $b_{1}=0.440$ and $a_{2}=0.910$. As above, these values were determined empirically using the RNA05 data set (Fig. 4c). The azimuthal component of the sugar-center location, $\xi$, is defined such that it maximizes the electron density along the $\overrightarrow{\mathrm{P}_{5^{\prime}} \mathrm{G}_{\text {candidate }}}$ and $\overrightarrow{\mathrm{G}_{\text {candidate }} \mathrm{P}_{3^{\prime}}}$ vectors.

These calculations define a single sugar center $G_{\text {candidate }}$ for each phosphate candidate. $\mathrm{G}_{\text {candidate }}$ is then used to define $\mathrm{Cl}_{\text {candidate }}^{\prime}$, the candidate $\mathrm{C}^{\prime}$ coordinate. To determine the location of $\mathrm{Cl}_{\text {candidate, a Cartesian coordinate system is defined }}^{\prime}$ with the origin at $\mathrm{G}_{\text {candidate }}$ (Fig. $5 a$ ). The $x$ axis of this coordinate system is defined as the bisector of $\overrightarrow{\mathrm{G}_{\text {candidate }} \mathrm{P}_{3^{\prime}}}$ and $\overrightarrow{\mathrm{G}_{\text {candidate }} \mathrm{P}_{5^{\prime}}}$ and the $y$ axis is defined as $\overrightarrow{\mathrm{G}_{\text {candidate }} \mathrm{P}_{3^{\prime}}} \times$ $\overrightarrow{\mathrm{G}_{\text {candidate }} \mathrm{P}_{5^{\prime}}}$. The $z$ axis is then defined as the cross product of the $y$ and $x$ axes. The coordinates of $\mathrm{Cl}_{\text {candidate }}^{\prime}$ are then defined as $(-1.036,0.202,-0.601)$ in this coordinate system. These coordinates were determined empirically using the RNA05 data set (Fig. 5b).

All phosphate candidates are then scored. When tracing the first nucleotide of a chain, the score for each candidate phosphate is given by

$$
s_{\text {overall }}=w_{\text {dist }}^{\prime} \ln \left(s_{\text {dist }}\right)+w_{\text {density }}^{\prime} \ln \left(s_{\text {density }}\right),
$$

where $w_{\text {dist }}^{\prime}=1, w_{\text {density }}^{\prime}=10$ and the individual $s$ terms are as defined below. Note that these candidate phosphates are the phosphates of nucleotide 2 , as the phosphate of nucleotide 1 was placed during the initial phosphate picking. When tracing any subsequent nucleotide, the score for each candidate phosphate is given by

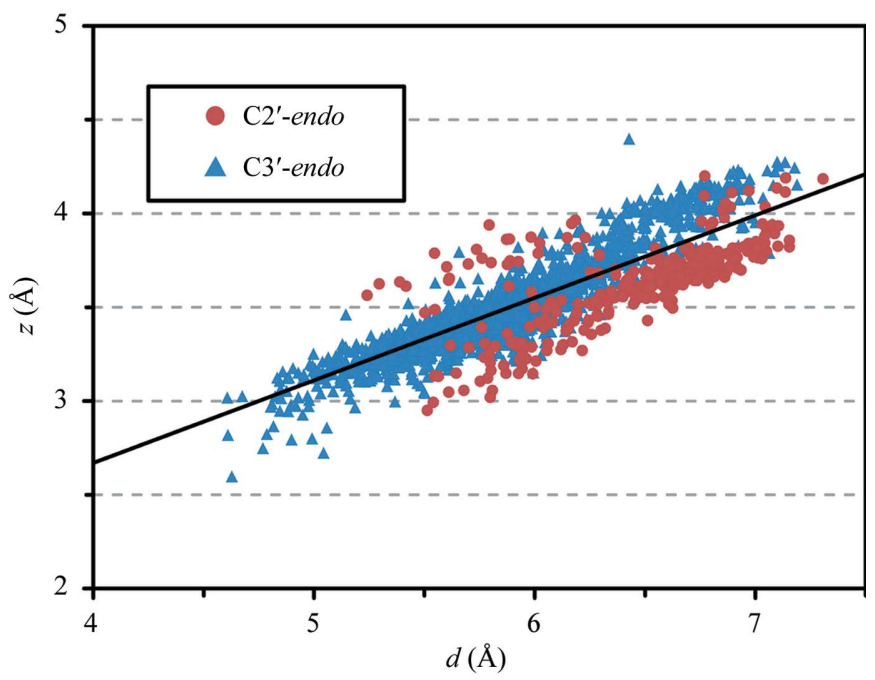

(c)

Figure 4

Two dimensions of the sugar-center location can be accurately predicted using only the coordinates of the $3^{\prime}$ and $5^{\prime}$ phosphates. $(a)$ The interphosphate distance $d$ is used to predict the radial and vertical components of the sugar-center location ( $r$ and $z$, respectively). (b) The radial component. Each point represents a nucleotide in the RNA05 data set, with sugar puckers as indicated. The quadratic regression (equation 1$)$ is shown. For this regression, $r^{2}=0.74$. (c) The vertical component. The linear regression (equation 3) is shown. For this regression, $r^{2}=0.78$. Note that the regressions in $(b)$ and $(c)$ were calculated using all data points, regardless of sugar pucker. 


$$
\begin{aligned}
s_{\text {overall }}= & w_{\text {dist }} \ln \left(s_{\text {dist }}\right)+w_{\text {angle_p }} \ln \left(s_{\text {angle_p }}\right) \\
& +w_{\text {angle_s }} \ln \left(s_{\text {angle_s }}\right)+w_{\text {density }} \ln \left(s_{\text {density }}\right),
\end{aligned}
$$

where $w_{\text {dist }}=5, w_{\text {angle_p }}=w_{\text {angle_s }}=1, w_{\text {dist }}=15$ and the individual $s$ terms are as defined below. Note that the values of all $w$ and $w^{\prime}$ terms were determined empirically.

The $s_{\text {dist }}$ score is the likelihood of finding two successive phosphates at distance $d$ in the RNA05 data set. The $s_{\text {angle_p }}$ score is the likelihood of finding three successive phosphates at an angle $\angle \mathrm{P}_{\text {prev }} \mathrm{P}_{\text {current }} \mathrm{P}_{\text {candidate }}$ in the RNA05 data set, where $\mathrm{P}_{\text {prev }}$ are the coordinates of the previous phosphate, $\mathrm{P}_{\text {current }}$ are the coordinates of the current phosphate and $\mathrm{P}_{\text {candidate }}$ are the coordinates of the candidate phosphate. The $s_{\text {angle_s }}$ score is the likelihood of finding successive $\mathrm{Cl}_{i-1}^{\prime}$, phosphate and $\mathrm{Cl}^{\prime}$ atoms at an angle $\angle \mathrm{Cl}_{\text {prev }}^{\prime} P_{\text {current }} \mathrm{Cl}_{\text {candidate }}^{\prime}$ in the RNA05 data set, where $\mathrm{C1}_{\text {prev }}^{\prime}$ are the coordinates of the previous $\mathrm{Cl}^{\prime}$ atom. The likelihood distributions for $s_{\text {dist }}, s_{\text {angle_p }}$ and $s_{\text {angle_s }}$ were calculated using kernel smoothing with a Gaussian kernel. The distributions themselves are provided as Supplementary Material ${ }^{\mathbf{1}}$. The $s_{\text {density }}$ score is the sum of the electron-density values at ten evenly spaced points along $\stackrel{\mathrm{P}_{\text {current }} \mathrm{G}_{\text {candidate }}}{\longrightarrow}$ ten evenly spaced points along $\overrightarrow{\mathrm{G}_{\text {candidate }} \mathrm{P}_{\text {candidate }}}, \mathrm{G}_{\text {candidate }}$ and $\mathrm{P}_{\text {candidate, with all electron- }}$ density values measured in e $\AA^{-3}$. Note that this $s_{\text {overall }}$ scoring metric shares elements with the scoring proposed by Gruene \& Sheldrick (2011).

The phosphate candidates are ranked based on their $s_{\text {overall }}$ scores. The $\mathrm{P}_{\text {candidate }}$ and associated $\mathrm{C}_{\text {candidate }}^{\prime}$ with the highest score are used as the basis for the default next nucleotide. Before this nucleotide is displayed, however, base coordinates are calculated. A single $5 \AA$ vector is used as a first approximation for the base, as this is roughly the distance between the $\mathrm{C1}^{\prime}$ and $\mathrm{C} 4$ atoms in pyrimidine (Fig. $6 a$ ). The start of this

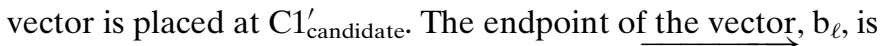
then placed to maximize the density along $\overrightarrow{\mathrm{Cl}_{\text {candidate }}^{\prime} \mathrm{b}_{\ell}}$ such that the angle between $\overrightarrow{\mathrm{Cl}_{\text {candidate }}^{\prime} \mathrm{b}_{\ell}}$ and the phosphate bisector is between $90^{\circ}$ and $180^{\circ}$, inclusive.

A pyrimidine base is then placed along the vector such that the $\mathrm{C}^{\prime}$ atom is located at $\mathrm{C}_{\text {candidate }}^{\prime}$ and the $\mathrm{C} 4$ atom is located along $\overrightarrow{\mathrm{Cl}_{\text {candidate }}^{\prime} \mathrm{b}_{\ell}}$. The base is then rotated about $\overrightarrow{\mathrm{C}_{\text {candidate }}^{\prime} \mathrm{b}_{\ell}}$ to maximize $s_{\text {base }}$, where

$$
s_{\text {base }}=s_{\text {ring_density }} s_{\text {pseudo- } \chi} \text {. }
$$

The $s_{\text {ring_density }}$ score is the sum of the electron-density values at all ring atoms measured in e $\AA^{-3}$ (note that the $\mathrm{O} 2$ and $\mathrm{N} 4 / \mathrm{O} 4$ atoms are ignored for this density fit). The $s_{\text {pseudo- } \chi}$ score serves to weight the base rotation towards typical syn, anti or high-anti values and is based on the pseudo- $\chi$ torsion angle, defined here for purines as the torsion of the phosphate, $\mathrm{C1}^{\prime}$, N9 and $\mathrm{N} 1$ atoms and for pyrimidines as the torsion of the phosphate, $\mathrm{C}^{\prime}, \mathrm{N} 1$ and $\mathrm{N} 3$ atoms. The $s_{\text {pseudo- } \chi}$ score is defined as the likelihood of finding the current pseudo- $\chi$ value in the RNA05 data set, with near-zero likelihood values replaced by

\footnotetext{
${ }^{1}$ Supplementary material has been deposited in the IUCr electronic archive (Reference: RR5014). Services for accessing this material are described at the back of the journal.
}

a floor value. This floor value prevents the exclusion of any strong fits to density owing to an unlikely pseudo- $\chi$ value. The likelihood distribution for $s_{\text {pseudo- } \chi}$ was calculated using kernel smoothing with a Gaussian kernel and the distribution is provided in the Supplementary Material ${ }^{\mathbf{1}}$. After maximizing $s_{\text {base, }}$, the newly built base is computationally mutated to the user-specified base type. For purines, this mutation aligns the ring atoms. For pyrimidines, this mutation aligns $\overrightarrow{\mathrm{C}_{\text {candidate }}^{\prime} \mathrm{b}_{\ell}}$ with the vector from $\mathrm{C}^{\prime}$ to the midpoint of the $\mathrm{C} 4-\mathrm{C} 5$ bond while maintaining the plane of the base (Fig. $6 a$ ). This ensures that the pyrimidine occupies roughly the same region of density as the purine.

If the crystallographer wishes to 'flip' a pyrimidine base between the anti and syn configurations, the base is rotated $180^{\circ}$ about a vector from $\mathrm{C} 1^{\prime}$ to the midpoint of the $\mathrm{C} 4-\mathrm{C} 5$ bond (Fig. 6b). This ensures that the flipped base is not moved out of the electron density, as would occur if the base were rotated about $\chi$. Purines, which are roughly symmetrical about the glycosidic bond, are simply rotated about $\chi$.

\subsection{Conformer prediction}

The initial publication of the consensus conformer library (Richardson et al., 2008) included eight 'wannabe' conformers that nearly satisfied the conformer-selection criteria. In the forthcoming update to the consensus conformer library, six of these 'wannabe' conformers have been promoted to full conformer status (Jain \& Richardson, 2011). RCrane includes these six promoted conformers $(2 \mathrm{~g}, 2 \mathrm{u}, 2 \mathrm{z}, 3 \mathrm{~g}, 5 \mathrm{n}$ and $5 \mathrm{r})$ in its predictions. Other than the inclusion of these new conformers, conformer prediction is carried out as described previously (Keating \& Pyle, 2010).

\subsection{Coordinate calculation}

The coordinate-calculation procedure used in RCrane is based on the previously reported minimization protocol (Keating \& Pyle, 2010). However, there are a number of important differences in the new protocol. In RCrane, Coot's built-in minimizer is used for coordinate calculation in place of a simplex minimizer. Coot's minimizer uses the conjugategradient minimization algorithm implemented in the GNU Scientific Library (Emsley et al., 2010; Galassi et al., 2009). Additionally, the sugar ring is no longer treated as a rigid element. Instead, strong restraints are placed on the $v_{0}, v_{1}$ and $v_{4}$ torsions, with the ideal values of these restraints being dependent upon the sugar pucker. The phosphate atom is also no longer fixed during minimization. Instead, a strong harmonic restraint is used to keep the atom close to its starting position. The minimization also now includes a weak term measuring the match between the structure and the electrondensity map. A weight of 10 is used for this term, which is significantly weaker than the default weight of 60 typically used in Coot.

\subsection{Rotamerization}

During rotamerization, conformer prediction is carried out as above. However, additional $5^{\prime}$ and $3^{\prime}$ heminucleotides are 
included during coordinate calculation. Torsion values from the existing structure are used as the torsion-restraint values for these heminucleotides. Such restraints are used for the $\alpha$, $\beta$ and $\gamma$ torsions of the $5^{\prime}$ heminucleotide and the $\varepsilon$ and $\zeta$ torsions of the $3^{\prime}$ heminucleotide. These restraints ensure proper geometry at the junction of the rotamerized nucleotides and the remainder of the structure.

\subsection{Density maps}

The results presented below make extensive use of two electron-density maps: the $3.1 \AA$ resolution group II intron map (Toor et al., 2008) and the $2.8 \AA$ resolution lysine riboswitch map (Garst et al., 2008). In the group II intron map the quality of the experimental phases was quite high, which meant that nonhelical regions could be reliably built directly into the experimentally phased map. As such, the experimentally phased map was used here. While the lysine riboswitch map was of higher resolution than the group II map, the experimental phases were of lower quality. As such, a modelphased map was calculated with REFMAC5 (Murshudov et al., 2011) using the coordinates of 118 helical nucleotides from the published structure. This mimicked a map that would be generated from crystallographic refinement after manually placing the helical regions of the structure.

\section{Results and discussion}

\subsection{Backbone tracing}

Building RNA structure using RCrane consists of three main tasks: backbone tracing, conformer prediction and coordinate calculation. Backbone tracing can be further divided into four steps: locating potential phosphates, locating sugar centers, locating $\mathrm{C1}^{\prime}$ atoms and locating bases. First,

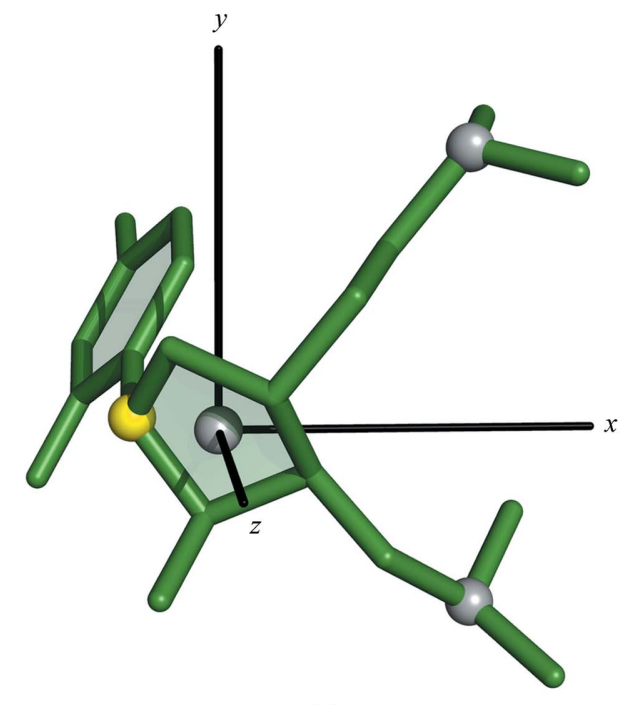

(a) potential phosphates are located by searching the electrondensity map for strong peaks of density that may correspond to phosphates (see \$3). In a previous study (Keating \& Pyle, 2010), we showed that accurate conformer predictions required phosphate coordinates within $1-1.5 \AA$ of their published locations. To test the newly developed phosphatesearch technique, we assessed how many phosphates within the group II intron (Toor et al., 2010) and lysine riboswitch (Garst et al., 2008) structures had nearby electron-density peaks. In the experimentally phased group II intron map, 290 of 388 phosphates $(75 \%)$ had a density peak within $1.0 \AA, 306$ phosphates $(79 \%)$ had a density peak within $1.5 \AA$ and 82
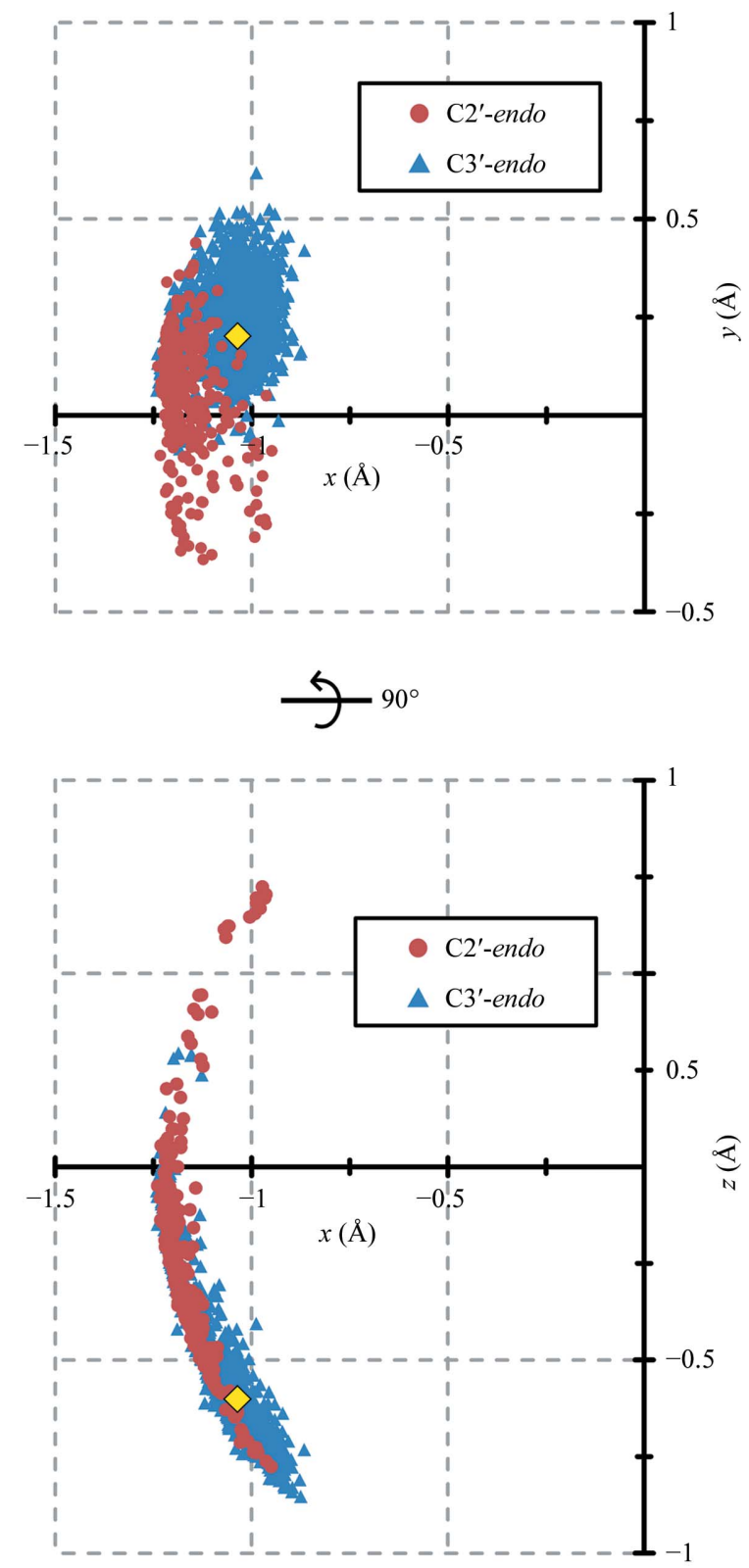

(b)

Figure 5

The coordinates of the $\mathrm{C}^{\prime}$ atom can be accurately determined after locating the sugar center. (a) The Cartesian coordinate system defined using the locations of the sugar center, $3^{\prime}$ phosphate and $5^{\prime}$ phosphate (see $\left.\$ 3\right)$. (b) Plots showing all $\mathrm{C}^{\prime}{ }^{\prime}$ atoms from the RNA05 data set relative to their respective sugar center, using the axes shown in (a) with sugar puckers as indicated. The top plot shows the $\mathrm{C}^{\prime}$ coordinates in the $x$ and $y$ axes, while the bottom plot shows the $x$ and $z$ axes. The mean $\mathrm{C}^{\prime}$ location is shown as a yellow diamond in both plots. During backbone tracing, $R C r a n e$ places new $\mathrm{C}^{\prime}$ atoms using this mean location. 
phosphates (21\%) had no peaks within $1.5 \AA$. Additionally, there were 524 density peaks that were further than $1.5 \AA$ from a published phosphate location. These peaks primarily corresponded to the published locations of ribose sugars, nucleoside bases, coordinated metal ions and structured water molecules. While this presents a large number of false-positive peaks, subsequent backbone-tracing steps serve to filter out these false positives and help the user to select peaks that correspond to true phosphate locations. The results were similar when using the model-phased map (see \$3) of the lysine riboswitch. In this map, 118 of 161 phosphates $(73 \%)$ had a peak within $1.0 \AA$, no additional phosphates had a peak within $1.5 \AA$, 43 phosphates $(27 \%)$ had no peak within $1.5 \AA$ and 410 peaks were further than $1.5 \AA$ from a published phosphate location.

When tracing the backbone, RCrane next calculates potential sugar center coordinates between pairs of potentially adjacent phosphates. To do this, the $r$ and $z$ coordinates of the sugar center are first predicted using the interphosphate distance (Fig. 4). These two values are calculated using regressions (see \$3) determined from the RNA05 data set (Richardson et al., 2008). Despite the simplicity of these calculations, the sugar center can be accurately located in the $r$ and $z$ dimensions. For nucleotides in RNA05, the average error in the $r$ and $z$ dimensions is $0.11 \AA$. These regressions are more accurate for $\mathrm{C}^{\prime}$-endo nucleotides than for $\mathrm{C}^{\prime}$-endo nucleotides. For $\mathrm{C}^{\prime}$-endo nucleotides in RNA05 the average error is $0.09 \AA$, while for $\mathrm{C} 2^{\prime}$-endo nucleotides the average error is $0.39 \AA$.

It should be noted that the relationship between $d$ and $r$ shown in Fig. 4(b) is best described by two separate linear regressions: one for the $\mathrm{C}^{\prime}$-endo nucleotides and one for the $\mathrm{C} 2^{\prime}$-endo nucleotides. However, during nucleotide tracing the sugar pucker is unknown. Therefore, these data were fitted using a single quadratic regression (see \$3) that adequately describes all nucleotides. Additionally, it should be noted that the correlation between $d$ and the sugar-center location is stronger than the correlation between $d$ and the $\mathrm{Cl}^{\prime}$ location, as the $\mathrm{Cl}^{\prime}$ coordinates are more sensitive to the sugar pucker (data not shown). This indicates that the sugar center may prove to be a useful anchor point for other modeling applications.

Next, the appropriate value of $\xi$ (Fig. $4 a$ ) is determined using the electron density (see $\$ 3$ ). This provides a single sugar-center location for each potential pair of adjacent phosphates. The $\mathrm{C1}^{\prime}$ coordinates can then be calculated from the sugar center using vector addition in a local Cartesian coordinate system (Fig. 5; see $\$ 3$ ). As above, these calculations were determined using the RNA05 data set (Richardson et al., 2008). For nucleotides in RNA05, the average error in estimating the $\mathrm{C}^{\prime}$ location from the sugar center is $0.17 \AA$. Again, these calculations are more accurate for $\mathrm{C}^{\prime}$-endo nucleotides than for $\mathrm{C2}^{\prime}$-endo nucleotides. For $\mathrm{C}^{\prime}$ '-endo nucleotides the average error is $0.14 \AA$, while for $\mathrm{C}^{2}$-endo nucleotides the average error is $0.46 \AA$.

As a further test of this $\mathrm{C}^{\prime}$-finding method, we assessed how accurately $\mathrm{Cl}^{\prime}$ atoms could be located in the group II intron and lysine riboswitch maps. First, all density peaks within $1.5 \AA$ of a published phosphate location were located as described above. For all pairs of density peaks that corresponded to two adjacent phosphates, the coordinates of these peaks and the electron-density map were used to predict a potential $\mathrm{C}^{\prime}$ location. This predicted location was then compared with the published $\mathrm{Cl}^{\prime}$ coordinates. In the group II intron density map this resulted in 265 predicted $\mathrm{Cl}^{\prime}$ locations, with $213(80 \%)$ of these predictions within $1.0 \AA$ of the published coordinates and $252(95 \%)$ within $1.5 \AA$ of the published coordinates. In the lysine riboswitch map there were 101 predicted $\mathrm{C}^{\prime}$ ' locations, with $100(99 \%)$ within $1.0 \AA$ of the published coordinates and all predicted locations within $1.2 \AA$ of the published coordinates.

After determining a $\mathrm{Cl}^{\prime}$ location, $R$ Crane next builds a base into the density adjacent to the $\mathrm{C}^{\prime}$ atom (see $\S 3$ ). As shown previously (Keating \& Pyle, 2010), conformer prediction is largely insensitive to imprecision in the base coordinates. However, accurate base positioning is clearly important for crystallographic refinement and interpretation of the resulting structure. The base-building method was tested using the group II intron and lysine riboswitch maps. For all $\mathrm{C1}^{\prime}$ coordinates that were predicted within $1.5 \AA$ of their published location, a base position was predicted and built using the appropriate base type (i.e. A, C, G or U). The r.m.s.d. was then calculated between the predicted and published base coordinates. In the group II intron map 252 bases were built. Of these bases, $123(49 \%)$ were within $1.0 \AA$ r.m.s.d. of their published coordinates and $213(85 \%)$ were within $2.0 \AA$ r.m.s.d. In the lysine riboswitch map 101 bases were built, with 74 bases $(74 \%)$ within 1.0 A r.m.s.d. and all bases within $1.9 \AA$ r.m.s.d.

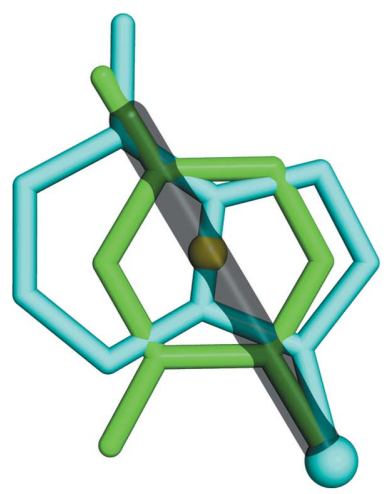

(a)

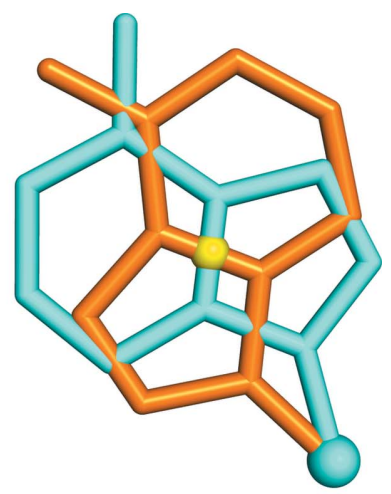

(b)
Figure 6

Positioning bases in density. (a) When placing bases into density, the nucleoside base is first approximated as a $5 \AA$ vector, shown here in transparent gray. This vector is anchored at the $\mathrm{C}^{\prime}$ candidate, which is shown as a cyan sphere. The vector is then replaced by a pyrimidine base, which may be computationally mutated to a purine if desired. To align the purine with the pyrimidine, the midpoint of the purine $\mathrm{C} 4-\mathrm{C} 5$ bond (yellow sphere) is aligned with the $5 \AA$ vector. (b) When flipping a pyrimidine between anti and syn configurations, the base is rotated about the $\mathrm{Cl}^{\prime}$ atom (cyan sphere) and the midpoint of the $\mathrm{C} 4-\mathrm{C} 5$ bond (yellow sphere). Flipping the base in this manner ensures that it is not moved out of the electron density. 


\subsection{Conformer prediction and coordinate calculation}

After backbone tracing is complete, RCrane must predict an appropriate conformer for each suite and then calculate atomic coordinates that match both the predicted conformer and the traced phosphate and base locations. Conformer prediction and coordinate calculation were carried out as previously described (Keating \& Pyle, 2010) with only minor modification (see $\$ 3$ ). The additional conformers considered during conformer prediction are rare and thus have a negligible impact on conformer-prediction accuracy (data not shown). The modifications to the coordinate-calculation procedure serve to dramatically speed up the minimization process, but do not produce substantially different coordinates. Both conformer prediction and coordinate calculation have previously been shown be highly accurate (Keating \& Pyle, 2010): one of the first three conformer predictions was correct $98 \%$ of the time and the first prediction was correct $84 \%$ of the time. Coordinate calculation built suites that matched the intended conformer $97.6 \%$ of the time, with many of the remaining $2.4 \%$ of suites containing only imperceptible differences to their target conformer (Keating \& Pyle, 2010). These steps are not explicitly further tested here.

\subsection{Building RNA using $R$ Crane}

Even when using RCrane, building an RNA structure into electron density is a complex process. To describe how RCrane fits into this procedure, we present here a general workflow for crystallographic RNA model building. It should be noted that this is a simplified and idealized description and that model building rarely proceeds entirely according to plan. The first step is to locate regions of the map that correspond to helical RNA. These regions can typically be identified even when the phase quality is poor. Additionally, RNA helical structure is highly regular, so these nucleotides can be accurately built even when the density is imprecise. This building can be performed using RCrane. Alternatively, if the experimental phases are so poor that locating individual phosphates and bases is difficult, then five to ten nucleotide stretches of idealized helical geometry can be manually docked into the density. Frequently, the crystallographer can then assign sequence to many of these newly built helices using the secondary structure, which is typically well characterized prior to crystallization.

After building the helices, a round of crystallographic refinement will dramatically improve the quality of the phases, resulting in a noticeably clearer density map. Next, nonhelical regions of the RNA can be built using RCrane. At this point, the length and sequence of many of these single-stranded regions is known. The building process then continues in an iterative manner, with improved phases helping to place new regions of structure and new regions of structure helping to improve phase quality.

\subsection{Test cases}

To further demonstrate the RCrane building process, we present a detailed description of building two nonhelical regions of RNA using RCrane: the GANC tetraloop of the group II intron (Keating et al., 2008) and an S-turn motif in the lysine riboswitch (Garst et al., 2008). This building was carried out without referring to the published coordinates. The GANC tetraloop (Fig. 3a) was built starting at the phosphate of nucleotide 367 and the structure was traced $5^{\prime}$ to $3^{\prime}$. When building the initial nucleotide, the third-choice phosphate location was used (i.e. the 'Next Phos' button was clicked twice), as RCrane initially began to trace in the direction of the helix rather than the tetraloop. When building the first nucleotide, RCrane has no existing structure or directional information to inform its initial backbone placement; hence, the first-choice phosphate location may be incorrectly placed. After this initial nucleotide, however, the default locations were used for all bases and phosphates. In total, seven nucleotides were traced.

After conformer prediction and coordinate calculation were carried out automatically, suite 372 was flagged as uncertain (colored orange) during alternate-conformer selection. The most likely conformer for this suite was $1 \mathrm{f}$, with a score of 0.40 , while the second most likely conformer was $1 \mathrm{a}$, with a score of 0.29. After examining the fit to density, this suite was changed to 1a. Additionally, 1a is the A-form helical conformer and therefore the most abundant backbone configuration. Conformer abundance is not considered during conformer prediction, but the abundance of 1a further supports its selection as an alternate conformer for this suite. No other suites were flagged as uncertain and so no other conformers were changed. The structure resulting from this building was highly accurate and closely matched the published GANC coordinates (Fig. 3a).

Building of the S-turn motif was started at the phosphate of nucleotide 20 and the structure was traced $5^{\prime}$ to $3^{\prime}$. Despite the model phasing (see $\$ 3$ ), this map was less precise than the group II intron map above, which led to erroneous default structure placement during backbone tracing. As a result, alternate phosphate locations were selected for most nucleotides (via the 'Next Phos' button). Additionally, nearly all base positions were manually adjusted by approximately $0.5 \AA$ to better fit the density and to improve stacking on the alreadybuilt $5^{\prime}$ nucleotides. As above, seven nucleotides were traced in total. After fully automated conformer prediction and coordinate calculation, suite 22 was flagged as uncertain (colored orange) during alternate-conformer selection. For this suite, 1a was the most likely conformer and was the best fit to the density, so the conformer was not changed. As with the tetraloop above, the resulting structure closely matched the published coordinates (Fig. 3b). Note that the base of nucleotide 24 was positioned incorrectly during backbone tracing by approximately $0.5 \AA$, leading to the slight discrepancy between the traced and published coordinates in this nucleotide.

From building into these and other maps, it is possible to make observations about the capabilities of RCrane. This building methodology appears to be more sensitive to phase quality than to map resolution. As a result, RCrane should be usable with X-ray crystallography density maps up to 
approximately $4 \AA$ resolution provided that the phase quality is sufficient to generate an interpretable map. However, with X-ray crystallography calculating accurate experimental phases typically becomes more difficult as the resolution worsens. This points to the possibility of using RCrane with cryo-electron microscopy (cryo-EM) data, as phase information can be directly and accurately measured from cryo-EM experiments. Additionally, the resolutions achieved via these experiments are continuously and rapidly improving (Seidelt et al., 2009; Yu et al., 2008). It is therefore possible that the RCrane methodology could soon be used to build a de novo all-atom structure into a cryo-EM density map. Further work in this area is planned.

Although there are relatively few tools designed specifically for crystallographic RNA model building, recent releases of $A R P / w A R P$ are able to build nucleic acids into electrondensity maps in an automated fashion (Hattne \& Lamzin, 2008). This building technique has been tested with proteinnucleic acid complexes, where it has been shown to aid in model building (Hattne \& Lamzin, 2008). In order to compare model building in RCrane with that in $A R P / w A R P$, we used $A R P / w A R P$ to build into the electron-density maps of the group II intron and lysine riboswitch. For the group II intron, ARP/wARP was able to automatically build 191 of 388 (49\%) nucleotides; however, the built nucleotides contained highly distorted geometry. Of the 191 built nucleotides, 182 (95\%) contained a steric clash greater than $0.4 \AA, 127$ (66\%) contained a steric clash greater than $1.0 \AA$ and 17 (9\%) contained a steric clash greater than $1.5 \AA$, as measured using MolProbity (Chen et al., 2010). Additionally, many of the bases were built in incorrect locations, with only 53 of the 191 built bases $(28 \%)$ within $2.0 \AA$ r.m.s.d. of their published locations. Furthermore, 35 nucleotides (18\%) were placed into density backwards (i.e. running $3^{\prime}$ to $5^{\prime}$ instead of $5^{\prime}$ to $3^{\prime}$ ). Owing to the severity of these issues, this automatically built structure would not provide a suitable starting point for further structure building or for crystallographic refinement.

For the lysine riboswitch map, $A R P / w A R P$ was able to build 120 of 161 nucleotides; however, the built structure again contained highly distorted geometry. Of the 120 built nucleotides, $91(76 \%)$ contained a steric clash greater than $0.4 \AA, 29(24 \%)$ contained a steric clash greater than $1.0 \AA$ and seven $(6 \%)$ contained a steric clash greater than $1.5 \AA$ (Chen et al., 2010). Base placement was dramatically improved relative to the group II intron building, as 91 of the 120 bases (76\%) were within $2.0 \AA$ r.m.s.d. of their published coordinates. However, 18 lysine riboswitch nucleotides $(15 \%)$ were placed into density backwards. Of the 120 built nucleotides, 106 corresponded to helical nucleotides that were used to calculate the map (see §3). The remaining 14 nucleotides consisted of eight segments, each of one to three nucleotides in length. All eight segments corresponded to nucleotides present in the published structure, indicating that they had been placed appropriately; however, the base planes of many of these nucleotides were approximately $45-90^{\circ}$ away from their published orientations. Additionally, ARP/wARP failed to build the remaining 29 nonhelical nucleotides. Therefore, even when using a model-phased map, extensive interactive building would be necessary after the use of $A R P / w A R P$.

These $A R P / w A R P$ results serve to highlight the difficulties in structure building for RNA crystallography. At first glance, the task of building RNA structure into an RNA-only density map and the task of building RNA structure into a proteinRNA complex density map seem highly similar. However, $A R P / w A R P$ struggles with the RNA-only maps tested here, even though it is perfectly capable of building into proteinRNA complex maps (Hattne \& Lamzin, 2008). This may, at least in part, be a consequence of phase quality. Protein structure can be built into protein-RNA complex maps first and then used to calculate accurate model phases. These model phases dramatically improve the quality of the RNA regions of the map. Particularly when there are far more amino acids than nucleotides in the complex, the complete protein structure is likely to provide significantly more phasing power than placing helices into an RNA-only map. As such, when building into RNA-only density maps, approaches such as $R$ Crane remain necessary.

\section{Conclusions}

RNA model building is a complex and involved process, as shown by the difficulties experienced with $A R P / w A R P$ when building into RNA-only density maps. However, RCrane allows a crystallographer to build RNA structure quickly and accurately by partially automating the model-building process, even when working with low-resolution or intermediateresolution data. This program assists a crystallographer in locating bases and phosphates within electron density. From this basic backbone trace, $R$ Crane can automatically construct a highly accurate all-atom model. RCrane works within Coot, thus providing an easy-to-use GUI interface that most crystallographers are already familiar with. RCrane is freely available from http://pylelab.org/software and runs on Windows, Linux and OS X. RCrane will also be included with all Coot installations starting with Coot 0.7 and will be accessible via the Extensions menu. This program is still under active development and continued improvement to the software is planned. A tutorial video is also available from http:// pylelab.org/software. Additionally, tests of RCrane with cryoEM data are planned. As this building technique appears to be more sensitive to poor phase quality than to low resolution, it is possible that the RCrane methodology could soon be used to build all-atom structure into cryo-EM density maps.

The authors wish to thank Paul Emsley and Bernhard Lohkamp for assistance with Coot programming. We also thank Rob Batey, Francis Reyes, Gary Kapral, Alfonso Mondragón, Nick Reiter and Gregor Blaha for initial testing of RCrane. We thank Jane Richardson, Laura Murray, Paul Emsley and Tom Terwilliger for numerous helpful discussions and suggestions during the development of this program. We thank Rob Batey, Francis Reyes, Alfonso Mondragón and Andrey Krasilnikov for providing experimentally phased 
maps for internal testing of RCrane, as well as Swati Jain and Jane Richardson for providing data on the promoted 'wannabe' conformers. This work was supported by the Howard Hughes Medical Institute and National Institutes of Health grant RO1 GM50313 to AMP.

\section{References}

Ban, N., Nissen, P., Hansen, J., Moore, P. B. \& Steitz, T. A. (2000). Science, 289, 905-920.

Batey, R. T., Gilbert, S. D. \& Montange, R. K. (2004). Nature (London), 432, 411-415.

Cao, S. \& Chen, S.-J. (2005). RNA, 11, 1884-1897.

Chen, V. B., Arendall, W. B., Headd, J. J., Keedy, D. A., Immormino, R. M., Kapral, G. J., Murray, L. W., Richardson, J. S. \& Richardson, D. C. (2010). Acta Cryst. D66, 12-21.

Cowtan, K. (2006). Acta Cryst. D62, 1002-1011.

Emsley, P., Lohkamp, B., Scott, W. G. \& Cowtan, K. (2010). Acta Cryst. D66, 486-501.

Galassi, M., Davies, J., Theiler, J., Gough, B., Jungman, G. Alken, P., Booth, M. \& Rossi, F. (2009). GNU Scientific Library Reference Manual, 3rd ed. Network Theory Ltd.

Garst, A. D., Héroux, A., Rambo, R. P. \& Batey, R. T. (2008). J. Biol. Chem. 283, 22347-22351.

Gruene, T. \& Sheldrick, G. M. (2011). Acta Cryst. A67, 1-8. Hattne, J. \& Lamzin, V. S. (2008). Acta Cryst. D64, 834-842. Jain, S. \& Richardson, J. S. (2011). Personal communication. Jonikas, M. A., Radmer, R. J., Laederach, A., Das, R., Pearlman, S., Herschlag, D. \& Altman, R. B. (2009). RNA, 15, 189-199.

Keating, K. S. \& Pyle, A. M. (2010). Proc. Natl Acad. Sci. USA, 107, $8177-8182$.
Keating, K. S., Toor, N. \& Pyle, A. M. (2008). J. Mol. Biol. 383, 475-481.

Langer, G., Cohen, S. X., Lamzin, V. S. \& Perrakis, A. (2008). Nature Protoc. 3, 1171-1179.

Lovell, S. C., Word, J. M., Richardson, J. S. \& Richardson, D. C. (2000). Proteins, 40, 389-408.

Murshudov, G. N., Skubák, P., Lebedev, A. A., Pannu, N. S., Steiner, R. A., Nicholls, R. A., Winn, M. D., Long, F. \& Vagin, A. A. (2011). Acta Cryst. D67, 355-367.

Richardson, J. S., Schneider, B., Murray, L. W., Kapral, G. J., Immormino, R. M., Headd, J. J., Richardson, D. C., Ham, D., Hershkovits, E., Williams, L. D., Keating, K. S., Pyle, A. M., Micallef, D., Westbrook, J. \& Berman, H. M. (2008). RNA, 14, 465-481.

Sarver, M., Zirbel, C. L., Stombaugh, J., Mokdad, A. \& Leontis, N. B. (2008). J. Math. Biol. 56, 215-252.

Seidelt, B., Innis, C. A., Wilson, D. N., Gartmann, M., Armache, J. P., Villa, E., Trabuco, L. G., Becker, T., Mielke, T., Schulten, K., Steitz, T. A. \& Beckmann, R. (2009). Science, 326, 1412-1415.

Selmer, M., Dunham, C. M., Murphy, F. V., Weixlbaumer, A., Petry, S., Kelley, A. C., Weir, J. R. \& Ramakrishnan, V. (2006). Science, 313, 1935-1942.

Terwilliger, T. C. (2003). Acta Cryst. D59, 38-44.

Toor, N., Keating, K. S., Fedorova, O., Rajashankar, K., Wang, J. \& Pyle, A. M. (2010). RNA, 16, 57-69.

Toor, N., Keating, K. S., Taylor, S. D. \& Pyle, A. M. (2008). Science, 320, 77-82.

Wadley, L. M., Keating, K. S., Duarte, C. M. \& Pyle, A. M. (2007). J. Mol. Biol. 372, 942-957.

Wang, X., Kapral, G., Murray, L., Richardson, D., Richardson, J. \& Snoeyink, J. (2008). J. Math. Biol. 56, 253-278.

Yu, X., Jin, L. \& Zhou, Z. H. (2008). Nature (London), 453, 415-419. 